\title{
CLINICAL RESEARCH ARTICLE Prenatal exposure to perfluoroalkyl substances and adipocytokines: the HOME Study
}

\author{
Catherine O. Buck ${ }^{1}$, Melissa N. Eliot ${ }^{2}$, Karl T. Kelsey ${ }^{2}$, Antonia M. Calafat ${ }^{3}$, Aimin Chen $^{4}$, Shelley Ehrlich ${ }^{4,5}$, Bruce P. Lanphear ${ }^{6}$ and \\ Joseph M. Braun ${ }^{2}$
}

OBJECTIVE: Gestational perfluoroalkyl substances exposure has been associated with decreased birthweight. We determined if gestational perfluoroalkyl substances exposure was associated with fetal metabolic markers using data from the HOME Study, a prospective birth cohort of pregnant women and their children in Cincinnati, Ohio.

METHODS: Maternal serum concentrations of perfluorooctanoic acid (PFOA), perfluorooctane sulfonic acid (PFOS),

perfluorononanoic acid, and perfluorohexane sulfonic acid were quantified. We measured neonatal adipocytokine (leptin and adiponectin) concentrations in umbilical cord serum, and estimated percent differences with a 2-fold increase in maternal perfluoroalkyl substances concentrations among 230 mother-infant pairs.

RESULTS: Median maternal serum PFOA and PFOS concentrations were $5.6 \mathrm{ng} / \mathrm{mL}$ and $14 \mathrm{ng} / \mathrm{mL}$, respectively. Leptin was positively correlated with infant birthweight $(p<0.001)$. There were no statistically significant associations between maternal perfluoroalkyl substances and neonatal adipocytokine concentrations; each 2-fold increase in PFOA was associated with a nonsignificant increase in leptin $(5 \% ; 95 \% \mathrm{Cl}$ : $-10,22)$ and adiponectin (7\%; 95\% Cl: $-4,19)$.

CONCLUSION: Despite known associations with reduced birthweight, gestational serum perfluoroalkyl substances concentrations were not associated with neonatal adipocytokine concentrations. Further exploration of pathways of perfluoroalkyl substances associated changes in birthweight may help identify biomarkers that could be used to identify at-risk populations and develop interventions.

Pediatric Research (2018) 84:854-860; https://doi.org/10.1038/s41390-018-0170-1

\section{INTRODUCTION}

Perfluoroalkyl substances are a class of man-made endocrine disrupting chemicals used in some packaging materials, food containers, fire-fighting foams, industrial surfactants, and stainand water-resistant coatings (e.g., carpets, upholstery, and apparel). ${ }^{1}$ While diet is the primary source of perfluoroalkyl substances exposure in humans, exposure through contaminated drinking water is widespread in communities in the United States. $^{2,3}$ Greater than 6 million U.S. residents are at risk of potentially harmful exposure to some perfluoroalkyl substances from drinking water. ${ }^{3}$ Because of their strong carbonfluoride bonds, perfluoroalkyl substances are resistant to chemical, thermal, and biological degradation, resulting in accumulation in tissues and biological half-lives ranging from 3.8 to 7.3 years. ${ }^{1,4}$ Four specific perfluoroalkyl substances, perfluorooctanoic acid (PFOA), perfluorooctane sulfonic acid (PFOS), perfluorononanoic acid (PFNA), and perfluorohexane sulfonic acid (PFHxS) have been detected almost universally in the serum of pregnant women and children. ${ }^{5}$ Prenatal perfluoroalkyl substances exposure has been associated with decreased birthweight, ${ }^{6,7}$ increased "catch-up" growth, and excess adiposity and risk of obesity in childhood and adulthood..$^{9-12}$ The mechanism of these associations is unknown, but some studies suggest that perfluoroalkyl substances may disrupt metabolism and adipogenesis. ${ }^{13,14}$

In vitro, perfluoroalkyl substances can activate peroxisome proliferator-activated receptor (PPAR) $a$ and $\gamma_{1}^{13}$ which may in turn affect lipid metabolism and adipocyte differentiation, respectively. ${ }^{14}$ For example, gestational PFOA exposure has been associated with increased leptin and subsequent obesity in adults in both animal models and human studies. ${ }^{15}$ Leptin and adiponectin are adipocytokines released by adipocytes, have roles in both energy homeostasis and glucose metabolism, and are associated with infant birthweight, weight gain, and risk of childhood obesity. ${ }^{16-19}$ Other endocrine and metabolism disrupting chemicals, such as persistent organic pollutants, have also been shown to increase adipogenesis via similar mechanisms. ${ }^{20}$

However, there are limited data about gestational perfluoroalkyl substances exposure and fetal markers of metabolism. Two studies have investigated the relationship between maternal perfluoroalkyl substances and neonatal adipocytokines, with conflicting results. One study reported no association between maternal plasma perfluoroalkyl substances exposure and neonatal adipocytokine concentrations, ${ }^{21}$ while the other reported a positive correlation between maternal serum PFOS concentrations and neonatal adiponectin concentrations. ${ }^{22}$

${ }^{1}$ Department of Pediatrics, Warren Alpert Medical School of Brown University, Providence, RI, USA; ${ }^{2}$ Department of Epidemiology, Brown University School of Public Health, Providence, RI, USA; ${ }^{3}$ Division of Laboratory Sciences, National Center for Environmental Health, Centers for Disease Control and Prevention, Atlanta, GA, USA; ${ }^{4}$ Department of Environmental Health, University of Cincinnati, Cincinnati, $\mathrm{OH}, \mathrm{USA} ;{ }^{5}$ Division of Biostatistics and Epidemiology, Cincinnati Children's Hospital Medical Center, Cincinnati, OH, USA and ${ }^{6}$ Faculty of Health and Sciences, Simon Fraser University, Burnaby, Canada

Correspondence: Catherine O. Buck (catybuck@gmail.com)

Received: 27 February 2018 Revised: 7 August 2018 Accepted: 13 August 2018

Published online: 13 September 2018 
The objective of this study was to further investigate the association between gestational serum perfluoroalkyl substances concentrations and umbilical cord serum adipocytokine concentrations in a prospective cohort of 230 pregnant women and their infants. Given the previously observed associations between perfluoroalkyl substances exposure and fetal growth, we hypothesized that neonates with higher perfluoroalkyl substances exposure would have lower neonatal leptin and adiponectin concentrations. Understanding the association between perfluoroalkyl substances exposure and metabolism biomarkers, such as adipocytokines, may uncover potential routes of intervention to prevent the development of childhood obesity.

\section{METHODS}

Study participants

For this study, we used data from the Health Outcomes and Measures of the Environment (HOME) Study, an ongoing prospective pregnancy and birth cohort originally designed to investigate the effect of early life exposure to toxic chemicals on children's growth and development. We recruited pregnant women prior to $16 \pm 3$ weeks gestation from nine prenatal clinics in the Cincinnati, Ohio region between March 2003 and January 2006. Eligible women were at least 18 years of age, English speaking, without diabetes, cancer, HIV infection or bipolar disorder, not taking thyroid or seizure medications, living in a home built prior to 1978 , and planning to deliver at one of three hospitals in the Cincinnati, $\mathrm{OH}$ region. Women provided written informed consent for both themselves and their child. The Institutional Review Boards (IRBs) of the delivery hospitals and Cincinnati Children's Hospital Medical Center (CCHMC) approved the study. The Centers for Disease Control and Prevention (CDC) and Brown University IRB relied on the determinations made by the CCHMC IRB.

Perfluoroalkyl substances exposure assessment

Given the long serum half-life of PFOA, PFOS, PFNA, and PFHxS, and their known ability to cross the placenta, ${ }^{4,23}$ we used maternal serum concentrations of perfluoroalkyl substances to estimate fetal exposure. We collected maternal blood at 16 and 26 weeks gestation, and within $48 \mathrm{~h}$ of delivery. We used the earliest available sample to reduce the potential impact of pregnancyrelated blood volume changes. After separating serum from whole blood, the samples were stored at $-80^{\circ} \mathrm{C}$ until analysis. Using online solid phase extraction coupled to high performance liquid chromatography-isotope dilution tandem mass spectrometry, we quantified serum concentrations of PFOA, PFOS, PFNA, and $\mathrm{PFHXS}^{24}$ Limits of detection (LODs) were: $0.082 \mathrm{ng} / \mathrm{mL}$ (PFNA), $0.1 \mathrm{ng} / \mathrm{mL}$ (PFHxS and PFOA) and $0.2 \mathrm{ng} / \mathrm{mL}$ (PFOS). We included reagent blanks and quality control (QC) samples in each analytic batch, with coefficients of variation for repeated QC measurements of approximately 6 percent. We detected the four perfluoroalkyl substances in all samples. Other perfluoroalkyl substances also assessed in the general population, such as perfluorodecanoic acid (PFDA) and perfluorooctane sulfonamide, had low variability between individuals in our sample. perfluoroalkyl substances concentrations were $\log _{2}$-transformed for all analyses.

Neonatal adipocytokine measurement

We measured leptin and adiponectin concentrations in umbilical cord serum samples obtained at delivery using an ELISA sandwich assay and BioTeck microtiter ELx 808 plate reader. The LODs were $0.8 \mathrm{ng} / \mathrm{mL}$ (leptin) and $<2 \mu \mathrm{g} / \mathrm{mL}$ (adiponectin). We included reagent blanks and QC samples in each analytic batch, with coefficients of variation for repeated QC measurements of approximately 11 and 17 percent for leptin and adiponectin, respectively. Adipocytokine concentrations were $\log _{2}$-transformed for all analyses.

\section{Covariates}

Trained research staff collected data on potential confounders of the relationship between serum perfluoroalkyl substances and neonatal adipocytokines using computer-assisted questionnaires and medical chart abstraction. Maternal sociodemographic factors included maternal age, race, education, income, insurance status, and employment status. Perinatal covariates included parity, maternal body mass index (BMI) at 16 weeks gestation, gestational diabetes, pregnancy induced hypertensive disorders, depression symptoms, and delivery type. Maternal dietary and lifestyle factors included alcohol use during pregnancy, serum cotinine concentration (a biomarker of tobacco exposure during pregnancy), prenatal vitamin intake, and frequency of fresh fruit and vegetable consumption. Neonatal factors included gestational age, gender, and gestational age and sex-standardized birthweight $z$-score. ${ }^{25}$

Statistical analysis

First, we described univariate characteristics of perfluoroalkyl substances and adipocytokine concentrations and examined correlations between leptin, adiponectin, and covariates. Next, we examined bivariate correlations between cord serum adipocytokines and maternal perfluoroalkyl substances serum concentrations. Using restricted cubic splines, we examined potential non-linear associations between maternal serum perfluoroalkyl substances concentrations, birthweight, and cord blood adipocytokines. Restricted cubic splines allow for non-linear doseresponse associations between exposure and outcome variables. ${ }^{26}$ We did not detect any significant non-linear associations between maternal serum perfluoroalkyl substances concentrations, birthweight, and cord blood adipocytokines using restricted cubic splines. Thus, we used linear regression to estimate the percent difference in neonatal leptin and adiponectin concentrations for each 2-fold increase in maternal perfluoroalkyl substances concentrations. Given their known associations with PFAS exposure $^{27,28}$ and neonatal adipocytokines, we adjusted for maternal age, race, education, income, parity, maternal BMI, tobacco exposure, delivery mode, and infant sex.

\section{Secondary analyses}

We performed several secondary analyses to further characterize the association between maternal perfluoroalkyl substances concentrations and neonatal adipocytokines. First, we examined the association between gestational perfluoroalkyl substances concentrations and differences in adipocytokines for neonates with similar adiposity. To do this, we used birthweight z-score as a proxy for neonatal adiposity, and calculated the residual deviation in leptin or adiponectin for a given birthweight z-score. We then estimated the association between perfluoroalkyl substances concentrations and these residuals, adjusting for maternal age, race, education, income, parity, maternal BMI, tobacco exposure, delivery mode, and infant sex. Next, because the leptin to adiponectin ratio has been associated with metabolic syndrome and insulin resistance in adults and children, ${ }^{29,30}$ we examined leptin to adiponectin ratio among neonates in relation to maternal perfluoroalkyl substances concentrations.

In addition, given previously observed sex differences in the association of prenatal perfluoroalkyl substances exposure with child health outcomes and neonatal leptin concentrations, we stratified our primary regression analysis by infant sex. ${ }^{15}$ Lastly, because of potential effects on fetal growth, we conducted sensitivity analyses excluding women with gestational diabetes, pregnancy induced hypertensive disorders, elevated depressive symptoms, and preterm birth in separate covariate adjusted regression models. ${ }^{17} \mathrm{~A}$ priori, we calculated that with a sample size of 200 and alpha of 0.05 , we would have $80 \%$ power to detect 
Prenatal exposure to perfluoroalkyl substances and adipocytokines: the...

CO Buck et al.

Table 1 Cord serum leptin $(\mathrm{ng} / \mathrm{mL})$ and adiponectin $(\mu \mathrm{g} / \mathrm{mL})$ and maternal serum PFOA and PFOS $(\mathrm{ng} / \mathrm{mL})$ concentrations among HOME study participants

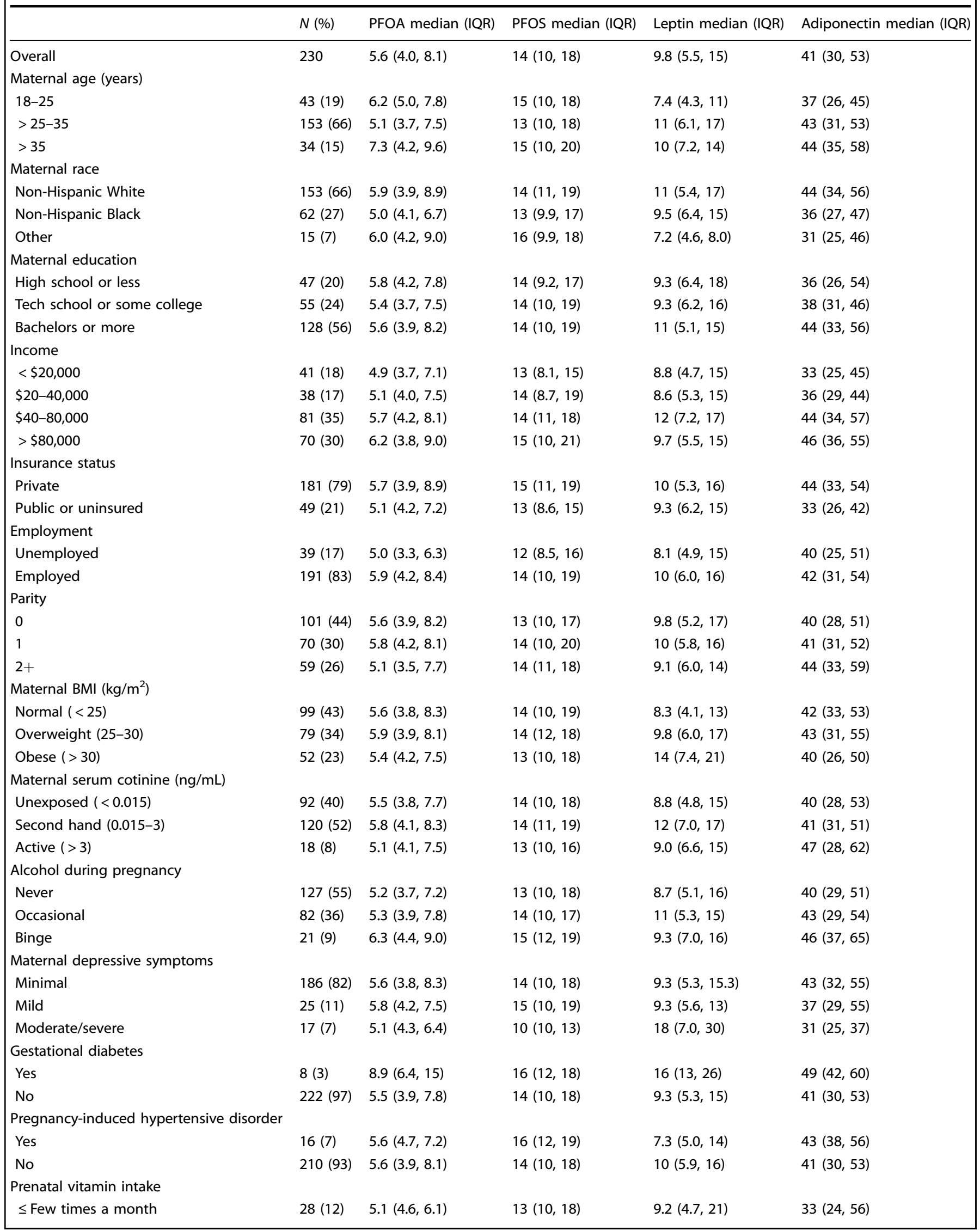




\begin{tabular}{|c|c|c|c|c|c|}
\hline & $N(\%)$ & PFOA median (IQR) & PFOS median (IQR) & Leptin median (IQR) & Adiponectin median (IQR) \\
\hline Weekly & $26(11)$ & $5.0(4.0,7.5)$ & $11(8.5,18)$ & $11(7.2,15)$ & $42(30,51)$ \\
\hline \multicolumn{6}{|c|}{ Fresh fruit and vegetable consumption } \\
\hline Monthly & $28(12)$ & $5.9(3.6,7.6)$ & $13(8.9,18)$ & $9.1(7.1,14)$ & $35(26,51)$ \\
\hline More than once a day & $48(21)$ & $5.2(3.7,8.5)$ & $14(10,18)$ & $12(7.5,17)$ & $41(32,48)$ \\
\hline \multicolumn{6}{|l|}{ Delivery mode } \\
\hline Vaginal & $165(72)$ & $5.3(3.8,7.6)$ & $14(10,18)$ & $9.3(5.5,15)$ & $43(31,55)$ \\
\hline Caesarian section & $65(28)$ & $6.1(4.2,9.0)$ & $14(10,19)$ & $12(5.3,17)$ & $40(27,48)$ \\
\hline \multicolumn{6}{|l|}{ Infant sex } \\
\hline Term ( $\geq 37$ weeks) & $215(94)$ & $5.5(3.9,8.1)$ & $14(10,18)$ & $11(6.6,16)$ & $42(32,54)$ \\
\hline \multicolumn{6}{|l|}{ Infant birth weight z-score } \\
\hline SGA ( < 10th percentile) & $17(7)$ & $7.5(5.2,9.3)$ & $15(11,19)$ & $4.9(2.6,8.1)$ & $42(34,50)$ \\
\hline AGA (10th-90th percentile) & $183(80)$ & $5.4(3.7,7.8)$ & $14(10,18)$ & $9.0(5.4,15)$ & $42(30,53)$ \\
\hline LGA (> 90th percentile) & $30(13)$ & $5.8(4.2,9.0)$ & $12.2(9,17)$ & $15(14,26)$ & $42(31,52)$ \\
\hline
\end{tabular}

IQR Interquartile range, PFOA Perfluorooctanoic acid, PFOS Perfluorooctane sulfonic acid, BMI Body Mass Index, SGA Small for gestational age, AGA Appropriate for gestational age, LGA Large for gestational age

a 0.2 standard deviation difference in cord blood adipocytokines for each standard deviation increase in serum perfluoroalkyl substances concentrations.

\section{RESULTS}

Among the 401 women initially enrolled in the HOME Study, we analyzed data for 230 mother-infant pairs who had complete data. We excluded those with multiples or stillbirths $(N=12,3 \%)$ and infants with chromosomal or genetic abnormalities $(N=2,<1 \%)$. We also excluded neonates who had insufficient cord serum for adipocytokine measurement $(N=94,23 \%)$, missing maternal serum perfluoroalkyl substances concentrations $(N=52,13 \%)$, and missing covariate information $(N=11,3 \%)$. Women in the HOME Study (Table 1) were predominantly college educated $(56 \%)$, white $(66 \%)$, and had private health insurance $(79 \%)$. Most infants were born at term (94\%) and had birthweight z-scores between the $10^{\text {th }}$ and $90^{\text {th }}$ percentiles (80\%). Covariates of women included in the study did not qualitatively differ from the full cohort of HOME Study participants (Supplemental Table S1 (online)).
Median maternal serum PFOA and PFOS concentrations were $5.6 \mathrm{ng} / \mathrm{mL}$ and $14 \mathrm{ng} / \mathrm{mL}$, respectively (Table 1). Median PFOA concentrations among women in our sample were higher than concentrations in pregnant women of the U.S. National Health and Nutrition Examination Survey (NHANES) between 2003 and 2006 $(2 \mathrm{ng} / \mathrm{mL}) .{ }^{15}$ Concentrations of the other perfluoroalkyl substances in our sample were similar to those among women in NHANES.

Among neonates in the study, median cord serum leptin and adiponectin were $9.8 \mathrm{ng} / \mathrm{mL}$ and $42 \mu \mathrm{g} / \mathrm{mL}$, respectively (Supplemental Table S2 (online)). Neonatal adiponectin and leptin were weakly positively correlated (Pearson correlation coefficient 0.25 , $p<0.001)$. Cord blood leptin was positively correlated with birthweight z-score (Pearson correlation coefficient $0.44, p<$ 0.001 ) and maternal BMI (Pearson correlation coefficient $0.27, p<$ 0.001 ). Unlike leptin, adiponectin was not significantly correlated with either birthweight or maternal BMI.

In adjusted models, the association between maternal serum perfluoroalkyl substances concentrations and neonatal adipocytokine concentrations were not statistically significant (Table 2). For instance, each 2-fold increase in PFOA was associated with a non-significant increase in leptin $(5 \% ; 95 \% \mathrm{Cl}:-10,22)$ and

\begin{tabular}{|c|c|c|c|c|}
\hline Perfluoroalkyl substances & $\begin{array}{l}\text { Leptin }(95 \% \mathrm{Cl}) \text {, } \\
\text { unadjusted }\end{array}$ & $\begin{array}{l}\text { Leptin }(95 \% \mathrm{Cl}) \text {, } \\
\text { adjusted }^{\mathrm{a}}\end{array}$ & $\begin{array}{l}\text { Adiponectin }(95 \% \mathrm{Cl}) \text {, } \\
\text { unadjusted }\end{array}$ & $\begin{array}{l}\text { Adiponectin }(95 \% \mathrm{Cl}) \text {, } \\
\text { adjusted }^{\mathrm{a}}\end{array}$ \\
\hline PFOA & $6(-9,24)$ & $5(-10,22)$ & $5(-5,17)$ & $7(-4,19)$ \\
\hline PFOS & $2(-14,21)$ & $2(-14,20)$ & $8(-4,21)$ & $4(-8,17)$ \\
\hline PFNA & $6(-14,31)$ & $1(-18,24)$ & $1(-13,17)$ & $-4(-17,12)$ \\
\hline
\end{tabular}


Table 3 Adjusted $^{\mathrm{a}}$ percent change in neonatal serum leptin and adiponectin concentrations per 2-fold increase in maternal serum perfluoroalkyl substances concentrations, stratified by infant sex

\begin{tabular}{lllll}
\hline & \multicolumn{1}{c}{ Leptin $(95 \% \mathrm{Cl})$} & & \multicolumn{2}{c}{ Adiponectin $(95 \% \mathrm{Cl})$} \\
\cline { 2 - 3 } Perfluoroalkyl substances & Male & Female & & Male \\
\hline PFOA & $16(-9,47)$ & $-1(-18,6)$ & $7(-8,25)$ \\
PFOS & $19(-9,56)$ & $-10(-27,12)$ & $3(-13,22)$ & $7(-10,28)$ \\
PFNA & $50(6,112)^{*}$ & $-22(-39,0)^{*}$ & & $0(-20,24)$ \\
PFHxS & $1(-16,21)$ & $-10(-22,3)$ & $6(-5,19)$ & $-6(-26,17)$ \\
\hline
\end{tabular}

CI Confidence Interval, PFOA Perfluorooctanoic acid, PFOS Perfluorooctane sulfonic acid, PFNA Perfluorononanoic acid, PFHXS Perfluorohexane sulfonic acid ${ }^{a}$ Adjusted for maternal age, race, education, income, parity, maternal body mass index, and serum cotinine

${ }^{*}$ Sex-PFAS interaction term $p<0.01$

adiponectin (7\%; 95\% Cl: $-4,19)$. Adjusting for sex- and gestational-age specific birthweight z-score did not significantly change the regression estimates (results not shown), and thus was not included as a covariate in our primary analyses.

Secondary analyses

There were no significant associations between maternal serum perfluoroalkyl substances concentrations and neonatal adipocytokines after accounting for infant adiposity, approximated with birthweight z-scores (Supplemental Table S3 (online)). Similar to the primary analyses, each 2-fold increase in PFOA was associated with a $3 \%$ increase in leptin $(95 \% \mathrm{Cl}:-10,17)$ and a $7 \%$ increase in adiponectin (95\% Cl: $-4,19)$. There were no associations between maternal serum perfluoroalkyl substances concentrations and neonatal leptin to adiponectin ratio (Supplemental Table S4 (online)). For example, there was a non-significant decrease in leptin to adiponectin ratio for each 2 -fold increase in maternal PFOA (-6\%, 95\% Cl: $-34,35)$ and PFOS (-3\%, 95\% Cl: $-34,43)$.

When we stratified our analyses by infant sex, perfluoroalkyl substances concentrations were positively associated with leptin concentrations in male infants, but inversely associated with leptin concentrations in female infants (Table 3). Each 2-fold increase in maternal serum PFNA was associated with a $50 \%$ increase $(95 \% \mathrm{Cl}$ : $6,112)$ in leptin in males and a $22 \%$ decrease $(95 \% \mathrm{Cl}:-39,0)$ in females. The interaction term between perfluoroalkyl substances and infant sex was statistically significant $(p<0.01)$ for PFNA, but not for the other perfluoroalkyl substances $(p>0.10)$. Finally, our results were similar when women with pregnancy induced hypertensive disorders, gestational diabetes, depressive symptoms, or preterm delivery were excluded from the regression analyses.

\section{DISCUSSION}

In this prospective cohort of pregnant women and their infants, maternal serum PFOA, PFOS, PFNA, and PFHxS concentrations were not associated with neonatal serum adipocytokine concentrations. Despite this, epidemiologic and toxicological evidence suggests that gestational perfluoroalkyl substances exposure is associated with impaired fetal growth. 6,7 While we did not find evidence that neonatal adipocytokine concentrations, markers of fetal growth, were associated with perfluoroalkyl substances serum concentrations, there is a need to examine other biological pathways involved in the association of perfluoroalkyl substances with fetal growth and obesity risk in order to identify at-risk populations and develop interventions.

Our findings are consistent with other studies investigating the relationship between prenatal perfluoroalkyl substances exposure and markers of fetal metabolism. In a cohort of more than 1500 mother-infant pairs, Ashley-Martin et al. ${ }^{21}$ found no association between maternal perfluoroalkyl substances concentrations
(PFOA, PFOS, and PFHxS) and cord blood leptin and adiponectin concentrations, including with stratification by infant sex. In contrast, a cohort study from Japan ${ }^{22}$ reported that maternal serum PFOS, but not PFOA, was associated with cord serum adiponectin among 168 mother-infant pairs. That study did not report any differences with stratification by infant sex, and did not find any associations between PFOS or PFOA with neonatal leptin. Finally, Fleisch et al. ${ }^{10}$ found no association between maternal perfluoroalkyl substances concentrations (PFOA, PFOS, PFNA, PFHxS, and PFDA) during pregnancy and mid-childhood leptin and adiponectin concentrations. The literature varies in regards to which specific perfluoroalkyl substances are measured and reported, which is important as some perfluoroalkyl substances are substituted by less studied replacements.

Gestational PFOA exposure affects fetal growth in toxicologic studies. A meta-analysis of 21 animal studies reported a 0.023 gram decrease in birthweight per $\mathrm{mg} / \mathrm{kg}$ body weight increase in PFOA exposure in pregnant rodents. ${ }^{31}$ Similarly, two metaanalyses of 18 human studies estimated a 15 to 19 gram decrease in birthweight for every $1 \mathrm{ng} / \mathrm{mL}$ increase in maternal PFOA serum concentration. ${ }^{6,7}$ Epidemiological studies have also observed an association of prenatal exposure to some perfluoroalkyl substances with childhood growth, adiposity, and risk of obesity. In girls, Mora et al. ${ }^{11}$ found that maternal serum perfluoroalkyl substances concentrations (PFOA, PFOS, PFNA, and PFHxS) were positively associated with markers of adiposity in mid-childhood. In boys, Andersen et al. ${ }^{12}$ found that gestational PFOS and PFOA concentrations were associated with increased weight and $\mathrm{BMI}$ at age 12 months. In a Dutch cohort, Halldorsson et al. ${ }^{15}$ reported that gestational PFOA exposure was associated with increased BMI and waist circumference in females at age 20. Finally, in the HOME Study, we previously observed that maternal serum PFOA concentration was positively associated with adiposity in midchildhood and changes in BMI trajectories from age 2 to 8 years. ${ }^{9}$

While the epidemiologic literature has consistently shown an association of prenatal exposure to some perfluoroalkyl substances with infant birthweight and childhood adiposity, the mechanism for these associations is unknown. The present and prior findings do not strongly support the hypothesis that perfluoroalkyl substances-induced alterations in leptin or adiponectin are responsible for the potential effect of perfluoroalkyl substances on fetal or childhood growth. However, there are other possible biological pathways worth additional consideration given that perfluoroalkyl substances can cross the placenta. ${ }^{23}$ These include epigenetic modification, oxidative stress, or other endogenous hormone pathways such as the hypothalamicpituitary-adrenal axis. In a study of adults, PFOA and PFOS were associated with expression of cholesterol metabolism genes. ${ }^{32}$ Moreover, in a pilot study among HOME Study participants, maternal serum PFOA concentrations were positively associated with neonatal peripheral leukocyte DNA methylation patterns; 
leptin receptor and promoter genes were not among the top 20 genes that differed by maternal serum PFOA concentrations. ${ }^{33}$ In a recent study, newborn PFOS concentrations were associated with an increase in reactive oxygen species concentrations. ${ }^{34}$ In vitro studies also report that some perfluoroalkyl substances can affect the action of hydroxysteroid dehydrogenases, which may in turn increase cortisol concentrations to adversely affect fetal growth. ${ }^{35}$ Future molecular epidemiology studies could determine if these pathways are related to perfluoroalkyl substances exposure, as well as changes in fetal and childhood growth.

We found some evidence that infant sex modified the association between maternal serum PFOA, PFOS, PFNA, and $\mathrm{PFHxS}$ concentrations with neonatal serum leptin concentrations. This was statistically significant for PFNA, but not the other perfluoroalkyl substances. In a prior epidemiological study, prenatal PFOA concentrations were associated with increased leptin and decreased adiponectin in adult women, but not men. ${ }^{15}$ Adipocytokine profiles are known to differ by sex, and this may be related to differences in gonadal hormone concentrations across sexes. Perfluoroalkyl substances may interact with mechanisms affecting testosterone or estrogen to differentially affect adipocytokine production or metabolism across genders. ${ }^{36}$ Future studies should continue to consider sex-specific effects of perfluoroalkyl substances on fetal growth and childhood adiposity, as well as potential biological pathways.

Strengths of our study include its prospective design and extensive covariate information, allowing for adjustment of numerous potential confounders. Additionally, women in our study had higher gestational serum PFOA concentrations than some other studies, ${ }^{21,22}$ which might have enabled us to detect a potential association between PFOA and neonatal adipocytokine concentrations. For instance, two prior studies had median PFOA concentrations of 1.4 and $1.7 \mathrm{ng} / \mathrm{mL}$, as compared to $5.6 \mathrm{ng} / \mathrm{mL}$ in our study. ${ }^{21,22}$ In our cohort, gestational PFOA concentrations were higher in women who were older, more educated, and not of non-Hispanic Black race/ethnicity. ${ }^{28}$ Consistent with our results, prior studies have shown that perfluoroalkyl substances concentrations may differ by maternal race and parity. ${ }^{5,37}$ Behavioral and lifestyle factors may account for some of the variation in perfluoroalkyl substances concentrations associated with sociodemographic features in our cohort. There is variation in some sociodemographic characteristics of participants across this and prior studies (e.g., race/ethnicity), ${ }^{10,21}$ and it is unclear whether this might have influenced differences in results. Future studies could consider whether these factors modify the association between perfluoroalkyl substances exposure and fetal metabolism biomarkers.

Limitations of our study include the potential for confounding due to unmeasured factors, including maternal renal function. We were unable to correct for maternal glomerular filtration rate, which may affect perfluoroalkyl substances excretion, and could be correlated with fetal growth. ${ }^{38}$ Prior studies, however, have found that perfluoroalkyl substances related growth changes at birth cannot be fully explained by maternal renal function alone. ${ }^{7}$ Additionally, we did not adjust for maternal dietary factors, which may impact both perfluoroalkyl substances exposure and fetal growth, and in turn, markers of fetal metabolism. Lastly, we did not account for potential variations in adipocytokine concentrations in the perinatal period. Maternal leptin increases during pregnancy, and has been positively associated with infant birthweight, but not with neonatal leptin concentrations. ${ }^{39}$ Neonatal adipocytokines represent a single measurement, and may not be generalizable to maternal or fetal adipocytokine exposure during pregnancy or postnatally. When measured postnatally, leptin concentrations rapidly decline in association with physiologic weight loss. ${ }^{40}$ There is limited information regarding variations in adiponectin concentrations around delivery.

\section{CONCLUSIONS}

In this prospective cohort of pregnant women and their infants with higher serum PFOA concentrations than the general U.S. population, we did not find a statistically significant association of maternal serum PFOS, PFOA, PFNA, and PFHxS concentrations with neonatal cord blood adipocytokine concentrations. Further exploration of pathways of perfluoroalkyl substances associated changes in fetal growth may help identify biomarkers that could be used to identify at-risk populations and develop interventions in response to perfluoroalkyl substances exposure.

\section{DISCLAIMER}

The findings and conclusions in this report are those of the authors and do not necessarily represent the official position of the Centers for Disease Control and Prevention. Use of trade names is for identification only and does not imply endorsement by the CDC, the Public Health Service, or the U.S. Department of Health and Human Services.

\section{ACKNOWLEDGEMENTS}

We thank Kayoko Kato and Tao Jia for the PFAS measurements and Theresa Kenney for the adipocytokine measurements. This work was supported by NIEHS grants R01 ES025214, R01 ES020349, and P01 ES11261.

\section{AUTHOR CONTRIBUTIONS}

J.M.B. and C.O.B. conceived the idea for this manuscript, and C.O.B. wrote the first draft. M.N.E. conducted the statistical analysis. J.M.B. oversaw C.O.B. and the statistical analysis. J.M.B., A.C., and B.P.L. are co-PIs of the HOME Study and provided feedback on the manuscript. K.T.K., A.M.C., and S.E. are collaborators on the HOME Study and provided feedback on the manuscript.

\section{ADDITIONAL INFORMATION}

The online version of this article (https://doi.org/10.1038/s41390-018-0170-1) contains supplementary material, which is available to authorized users.

Competing interests: The authors declare no competing interests.

Publisher's note: Springer Nature remains neutral with regard to jurisdictional claims in published maps and institutional affiliations.

\section{REFERENCES}

1. Buck, R. C. et al. Perfluoroalkyl and polyfluoroalkyl substances in the environment: terminology, classification, and origins. Integr. Environ. Assess. Manag. 7, 513-541 (2011).

2. Fromme, H., Tittlemier, S. A., Volkel, W., Wilhelm, M. \& Twardella, D. Perfluorinated compounds-exposure assessment for the general population in Western countries. Int. J. Hyg. Environ. Health 212, 239-270 (2009).

3. Hu, X. C. et al. Detection of Poly- and Perfluoroalkyl Substances (PFASs) in U.S drinking water linked to industrial sites, military fire training areas, and wastewater treatment plants. Environ. Sci. Technol. Lett. 3, 344-350 (2016).

4. Olsen, G. W. et al. Half-life of serum elimination of perfluorooctanesulfonate, perfluorohexanesulfonate, and perfluorooctanoate in retired fluorochemical production workers. Environ. Health Perspect. 115, 1298-1305 (2007).

5. Jain, R. B. Estimation of the total concentration of perfluoroalkyl acids (PFAA) in human serum: Data from NHANES 2005-2012. Chemosphere 134, 387-394 (2015).

6. Johnson, P. I. et al. The Navigation Guide-evidence-based medicine meets environmental health: systematic review of human evidence for PFOA effects on fetal growth. Environ. Health Perspect. 122, 1028-1039 (2014).

7. Verner, M. A. et al. Associations of perfluoroalkyl substances (PFAS) with lower birth weight: an evaluation of potential confounding by glomerular filtration rate using a physiologically based pharmacokinetic model (PBPK). Environ. Health Perspect. 123, 1317-1324 (2015).

8. Maisonet, M. et al. Maternal concentrations of polyfluoroalkyl compounds during pregnancy and fetal and postnatal growth in British girls. Environ. Health Perspect. 120, 1432-1437 (2012).

9. Braun, J. M. et al. Prenatal perfluoroalkyl substance exposure and child adiposity at 8 years of age: the HOME study. Obesity 24, 231-237 (2016). 
10. Fleisch A. F., et al. Early life exposure to perfluoroalkyl substances and childhood metabolic function. Environ. Health Perspect. 125, 481-487 (2016).

11. Mora A. M., Oken E., Rifas-Shiman S. L., et al. Prenatal exposure to perfluoroalkyl substances and adiposity in early and mid-childhood. Environ. Health Perspect. 125, 467-473 (2016).

12. Andersen, C. S. et al. Prenatal exposures to perfluorinated chemicals and anthropometric measures in infancy. Am. J. Epidemiol. 172, 1230-1237 (2010).

13. Taxvig, C. et al. Differential effects of environmental chemicals and food contaminants on adipogenesis, biomarker release and PPAR gamma activation. Mol. Cell. Endocrinol. 361, 106-115 (2012).

14. Moseti D., Regassa A., Kim W. K. Molecular regulation of adipogenesis and potential anti-adipogenic bioactive molecules. Int. J. Mol. Sci. 17, 124-148 (2016).

15. Halldorsson, T. I. et al. Prenatal exposure to perfluorooctanoate and risk of overweight at 20 years of age: A Prospective Cohort Study. Environ. Health Perspect. 120, 668-673 (2012).

16. Karakosta, P. et al. Leptin levels in cord blood and anthropometric measures at birth: a systematic review and meta-analysis. Paediatr. Perinat. Epidemiol. 25, 150-163 (2011)

17. Karakosta, P. et al. Cord blood leptin levels in relation to child growth trajectories. Metab.-Clin. Exp. 65, 874-882 (2016).

18. Boeke, C. E. et al. Differential associations of leptin with adiposity across early childhood. Obesity 21, 1430-1437 (2013).

19. Mantzoros, C. S. et al. Cord blood leptin and adiponectin as predictors of adiposity in children at 3 years of age: a prospective cohort study. Pediatrics 123, 682-689 (2009)

20. Nadal, A., Quesada, I., Tuduri, E., Nogueiras, R. \& Alonso-Magdalena, P. Endocrinedisrupting chemicals and the regulation of energy balance. Nat. Rev. Endocrinol. 13, 536-546 (2017).

21. Ashley-Martin J., et al. Maternal concentrations of perfluoroalkyl substances and fetal markers of metabolic function and birth weight: the maternal-infant research on environmental chemicals (MIREC) study. Am. J. Epidemiol. 185,185-193 (2017).

22. Minatoya, M. et al. Association of prenatal exposure to perfluoroalkyl substances with cord blood adipokines and birth size: The Hokkaido Study on environment and children's health. Environ. Res. 156, 175-182 (2017).

23. Midasch, O., Drexler, H., Hart, N., Beckmann, M. W. \& Angerer, J. Transplacental exposure of neonates to perfluorooctanesulfonate and perfluorooctanoate: a pilot study. Int. Arch. Occup. Environ. Health 80, 643-648 (2007).

24. Kato, K., Basden, B. J., Needham, L. L. \& Calafat, A. M. Improved selectivity for the analysis of maternal serum and cord serum for polyfluoroalkyl chemicals. J. Chromatogr. A 1218, 2133-2137 (2011).
25. Oken, E., Kleinman, K. P., Rich-Edwards, J. \& Gillman, M. W. A nearly continuous measure of birth weight for gestational age using a United States national reference. BMC Pediatr. 3, 6 (2003).

26. Desquilbet, L. \& Mariotti, F. Dose-response analyses using restricted cubic spline functions in public health research. Stat. Med. 29, 1037-1057 (2010).

27. Kato, K. et al. Changes in serum concentrations of maternal poly- and perfluoroalkyl substances over the course of pregnancy and predictors of exposure in a multiethnic cohort of Cincinnati, Ohio Pregnant Women during 2003-2006. Environ. Sci. Technol. 48, 9600-9608 (2014).

28. Kingsley, S. L. et al. Variability and predictors of serum perfluoroalkyl substance concentrations during pregnancy and early childhood. Environ. Res. 165, 247-257 (2018).

29. Finucane, F. M. et al. Correlation of the leptin:adiponectin ratio with measures of insulin resistance in non-diabetic individuals. Diabetologia 52, 2345-2349 (2009).

30. Mihalopoulos, N. L. et al. Leptin:adiponectin ratio, and metabolic dysfunction in adolescents with obesity. South. Med. J. 110, 347-352 (2017).

31. Koustas, E. et al. The Navigation Guide-evidence-based medicine meets environmental health: systematic review of nonhuman evidence for PFOA effects on fetal growth. Environ. Health Perspect. 122, 1015-1027 (2014).

32. Watkins, D. J. et al. Associations between serum perfluoroalkyl acids and LINE-1 DNA methylation. Environ. Int. 63, 71-76 (2014).

33. Kingsley, S. L. et al. Maternal serum PFOA concentration and DNA methylation in cord blood: A pilot study. Environ. Res. 158, 174-178 (2017)

34. Liu, H. et al. Prenatal exposure to perfluoroalkyl and polyfluoroalkyl substances affects leukocyte telomere length in female newborns. Environ. Pollut. 235, 446-452 (2018)

35. Zhao, B. et al. The inhibition of human and rat 11 beta-hydroxysteroid dehydrogenase 2 by perfluoroalkylated substances. J. Steroid Biochem. Mol. Biol. 125, 143-147 (2011)

36. Lopez-Espinosa, M. J., Mondal, D., Armstrong, B. G., Eskenazi, B. \& Fletcher, T. Perfluoroalkyl substances, sex hormones, and insulin-like growth factor-1 at 6-9 years of age: a cross-sectional analysis within the C8 Health Project. Environ. Health Perspect. 124, 1269-1275 (2016).

37. Jain, R. B. Effect of pregnancy on the levels of selected perfluoroalkyl compounds for females aged 17-39 years: data from National Health and Nutrition Examination Survey 2003-2008. J. Toxicol. Environ. Health A 76, 409-421 (2013).

38. Morken N. H., Travlos G. S., Wilson R. E., Eggesbo M., Longnecker M. P. Maternal glomerular filtration rate in pregnancy and fetal size. PLOS ONE 9, e101897 (2014).

39. Misra, V. K., Straughen, J. K. \& Trudeau, S. Maternal serum leptin during pregnancy and infant birth weight: the influence of maternal overweight and obesity. Obesity 21, 1064-1069 (2013).

40. Marchini, G., Fried, G., Ostlund, E. \& Hagenas, L. Plasma leptin in infants: relations to birth weight and weight loss. Pediatrics 101, 429-432 (1998). 